\title{
Measuring nutritional knowledge using Item Response Theory and its validity in European adolescents
}

\author{
Thanise Sabrina Souza Santos ${ }^{1, *}$, Cristina Julian², Dalton Francisco de Andrade ${ }^{3}$, \\ Betzabeth Slater Villar ${ }^{1}$, Raffaela Piccinelli ${ }^{4}$, Marcela González-Gross ${ }^{5}$, \\ Fréderic Gottrand ${ }^{6}$, Odysseas Androutsos ${ }^{7}$, Mathilde Kersting ${ }^{8}$, Nathalie Michels 9 , \\ Inge Huybrechts ${ }^{10}$, Kurt Widhalm ${ }^{10}$, Dénes Molnár ${ }^{12}$, Ascensión Marcos ${ }^{13}$,
} Manuel J Castillo-Garzón ${ }^{14}$ and Luis A Moreno ${ }^{2}$ on behalf of the HELENA Study Group $†$ 'Public Health Faculty, University of São Paulo, Av. Dr Arnaldo 715, Cerqueira César, São Paulo - SP, 01246-904, Brazil: ${ }^{2}$ Growth, Exercise, NUtrition and Development (GENUD) Research Group, Universidad de Zaragoza, Instituto Agroalimentario de Aragón (IA2), Instituto de Investigación Sanitaria de Aragón (IIS Aragón) and Centro de Investigación Biomédica en Red de Fisiopatología de la Nutrición y la Obesidad (CIBEROBN), Zaragoza, Spain: ${ }^{3}$ Department of Informatics and Statistics, Federal University of Santa Catarina, Florianópolis, SC, Brazil: ${ }^{4}$ Council for Agricultural Research and Economics, Research Centre for Food and Nutrition, Rome, Italy: ${ }^{5}$ Department of Health and Human Performance, Universidad Politécnica de Madrid, Centro de Investigación Biomédica en Red de Fisiopatología de la Nutrición y la Obesidad (CIBEROBN), Madrid, Spain: ${ }^{6}$ Department of Pediatrics, Regional Hospital Center, University of Lille, Lille, France: ${ }^{7}$ Department of Nutrition and Dietetics, School of Health Sciences and Education, Harokopio University, Athens, Greece: ${ }^{8}$ Research Department of Child Nutrition, Pediatric University Clinic, Bochum, Germany: ${ }^{9}$ Department of Public Health, Ghent University, Ghent, Belgium: ${ }^{10}$ Nutritional Epidemiology Group (NEP), International Agency for Research on Cancer, Lyon, France: " 1 Division of Nutrition and Metabolism, Department of Pediatrics, Medical University of Vienna, Vienna, Austria: ${ }^{12}$ Department of Pediatrics, University of Pécs Medical School, Pécs, Hungary: ${ }^{13}$ Institute of Food Science, Technology and Nutrition, Spanish National Research Council (ICTAN-CSIC), Madrid, Spain: ${ }^{14}$ Department of Physiology, Medicine School, University of Granada, Granada, Spain

Submitted 20 January 2018: Final revision received 16 0ctober 2018: Accepted 22 0ctober 2018: First published online 3 December 2018

\begin{abstract}
Objective: To analyse the Nutritional Knowledge Test (NKT) using Item Response Theory (ITR) analysis and to assess the construct validity of the Nutritional Knowledge Scale (NKTS) and its associations with adolescent food group consumption and nutritional biomarkers.

Design: Cross-sectional study.

Setting: Multicentre investigation conducted in ten European cities.

Participants: Adolescents aged $12 \cdot 5-17 \cdot 5$ years ( $n$ 3215) who completed over $75 \%$ of the NKT.

Results: Factor analysis indicated that the NKT can be analysed with a onedimensional model. Eleven out of twenty-three items from the NKT presented adequate parameters and were selected to be included in the NKTS. Nutrition knowledge was positively associated with consumption of fruits, cereals, dairy products, pulses, meat and eggs, and fish, as well as with blood concentrations of vitamin C, $\beta$-carotene, $n$ - 3 fatty acids, holo-transcobalamin, cobalamin and folate; nutrition knowledge was negatively associated with intake of olives and avocado, alcohol and savoury snacks.

Conclusions: The NKTS assessed nutritional knowledge adequately and it is proposed as a new tool to investigate this subject in future studies.
\end{abstract}

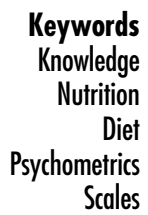

Keywords

Knowledge

Diet

Scales
Unhealthy diet contributes to nutrition- and obesity-related diseases, such as heart disease, cancer and type 2 dia-

$\dagger$ Members of the HELENA Study Group are listed in the Appendix. betes $^{(1)}$. One strategy to improve overall health and dietary habits is to increase knowledge about nutrition. Adolescence is a period in life during which nutritional education can help adolescents establish healthy eating habits and maintain them throughout life ${ }^{(2,3)}$. 
Nutritional knowledge is usually evaluated in healthy adolescents $^{(3-5)}$ with classical test theory ${ }^{(2)}$, based on the sum of correct answers from a true/false or multiplechoice questionnaire $^{(3-5)}$. The Nutritional Knowledge Test (NKT) was used in the Healthy Lifestyle in Europe by Nutrition in Adolescence (HELENA) study, a crosssectional and multicentre investigation. The HELENA study explored the level of nutritional knowledge among European adolescents and its potential determinants based on the percentage of correct answers ${ }^{(3)}$.

The classical test approach has some limitations. The characteristics of each item may vary across different populations. Thus, the validation of the questionnaire should be analysed again ${ }^{(2,6)}$. Item Response Theory (IRT) analysis provides information on the discrimination and difficulty of each item across different levels of the latent trait. The information of items remains constant even if the analyses are performed in other samples. In this regard, IRT analysis allows selection of items with a better distinction of the latent trait and acknowledges the level of the construct required by each item. Moreover, IRT scores are not only estimated from the sum of the correct answers but calculated based on a probabilistic model. Besides, IRT scores are also independent of the considered set of items ${ }^{(6)}$.

Therefore, IRT analysis may refine the measurement provided by the NKT, selecting items that better discriminate adolescent nutritional knowledge while characterizing the ability inherent on each level. The IRT scores calculated could also improve study of the relationship between nutritional knowledge and adolescent food intakes and biomarkers. Thus, the objectives of the present study were: (i) to analyse the NKT based on ITR analysis; and (ii) to assess the construct validity of the Nutritional Knowledge Scale (NKTS) and its associations with adolescent food group consumption and nutritional biomarkers. To address these objectives, nutritional knowledge was considered the latent trait on the IRT analysis encompassing energy metabolism, nutrient contents, oral health, food knowledge and nutritional terms.

\section{Methods}

\section{Study design and participants}

The HELENA study is a cross-sectional, multicentre investigation conducted in ten European cities: Vienna (Austria), Ghent (Belgium), Lille (France), Dortmund (Germany), Athens and Heraklion (Greece), Pécs (Hungary), Rome (Italy), Zaragoza (Spain) and Stockholm (Sweden) $^{(7)}$. Between October 2006 and December 2007, the HELENA study was carried out in a random cluster sample of 3528 European adolescents between 12.5 and 17.49 years old and stratified by geographical location, age and socio-economic level. To guarantee this stratification, school and class of adolescents by age group were considered. Overall, ten schools and fifteen to twenty classes (350-400 students) were selected in each city. The eligibility of each class within the selected schools was based on at least $70 \%$ of students agreeing to participate in the HELENA study. Blood samples were then obtained by randomly selecting one-third of the adolescents ( $n$ 1089) from the total sample ${ }^{(7)}$.

Exclusions from the HELENA study were performed $a$ posteriori when: adolescents presented simultaneous participation in another clinical trial; were $<12.5$ or $\geq 17.5$ years old; and/or had an acute infection less than one week before inclusion in the study. To process the IRT analyses, a total of 222 adolescents who did not complete more than $75 \%$ of the NKT on the first run of the test were excluded. In addition, ninety-one adolescents providing $100 \%$ of the answers as either right or wrong were also excluded from the current analyses because of the nonvariability of the latent trait, which could affect the development of the NKTS. In total, 313 adolescents did not meet the inclusion criteria for the current analyses. Therefore, the final sample comprised 3215 adolescents.

However, a different sample size was considered in the analyses. Those adolescents who had no information on dietary assessment and under-reported energy intake using the Goldberg et al. approach ${ }^{(8,9)}$ were excluded. This approach defines the minimum reasonable value of energy consumption that is compatible with energy balance, considering the level of physical activity ${ }^{(8,9)}$. In total, 487 adolescents under-reported energy intake. Moreover, for the mixed-model linear regression analyses, adolescents had to provide complete information on the corresponding confounding factors (age, gender, socio-economic level, maternal education level, centre and BMI). The final sample on the mixed model-linear regression analyses with consumption of food groups and biomarkers as the outcome variables included 1623 adolescents. The final sample for the mixed-model linear regression analyses with biomarkers as the outcome variable included 609 adolescents.

In each of the participating countries, the corresponding institution approved the HELENA study protocol and all participants and both parents or guardians provided their written consent ${ }^{(7,10)}$. Each participant was identified anonymously using a specific coding system which was included on the questionnaires and blood samples ${ }^{(10)}$.

The study design is further explained in Fig. 1.

\section{Sociodemographic characteristics}

Socio-economic status was evaluated with a self-reported questionnaire that collects data on living conditions, family structure, employment status of parents, parental occupation and educational level ${ }^{(11)}$. The Family Affluence Scale (FAS) index was calculated based on this questionnaire and was used as an indicator of adolescents' material affluence (reflecting the number of bedrooms, 


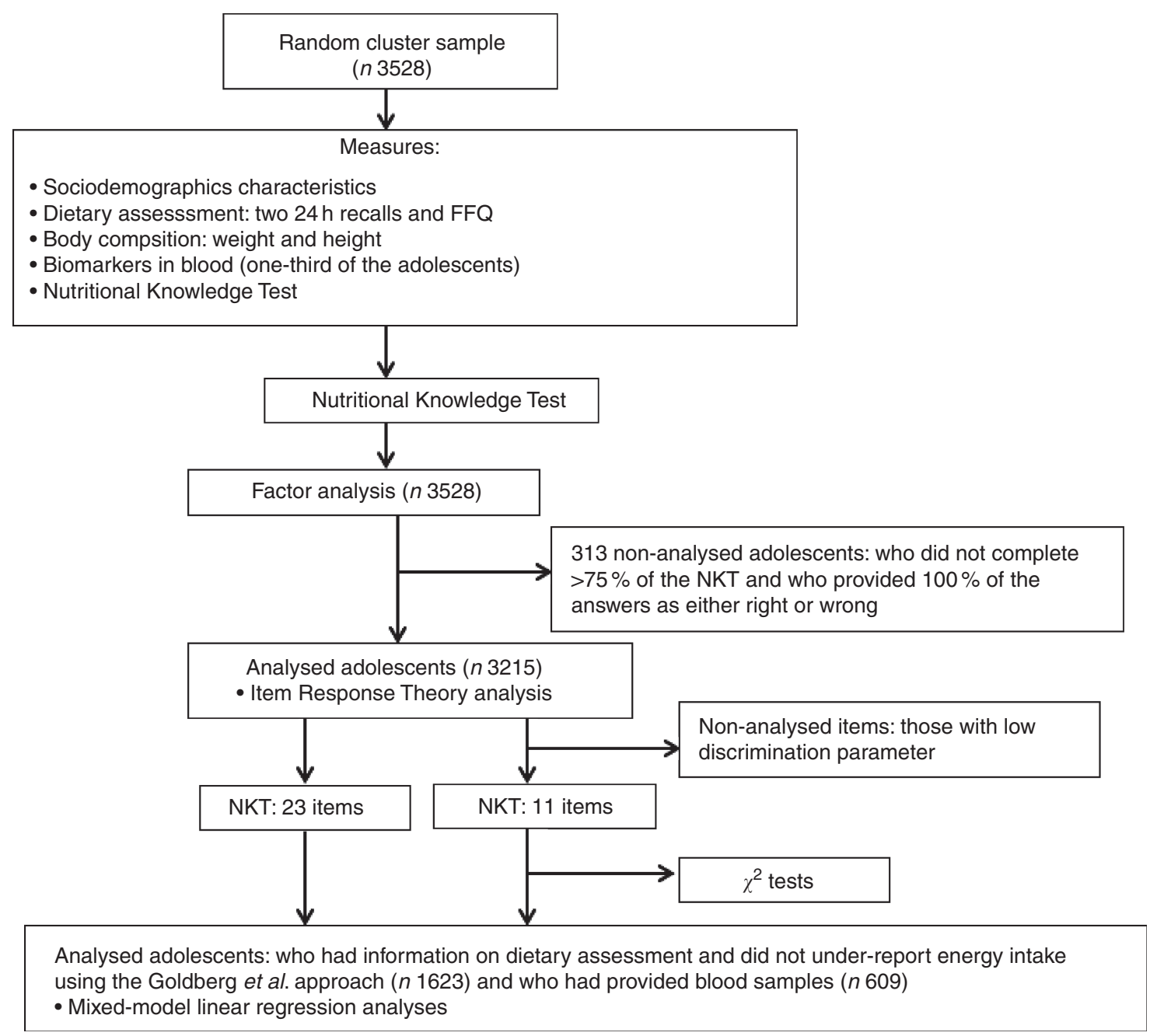

Fig. 1 Study design flowchart (NKT, Nutritional Knowledge Test; NKTS, Nutritional Knowledge Scale)

cars and computers possessed by the family and Internet access in their home). The scale ranged from 0 to 8 and was dichotomized into 'low familial wealth' (0-4) and 'high familial wealth' (5-8). Maternal education level was categorized into 'primary education', 'lower secondary education', 'higher secondary education' and 'university degree $^{,(12)}$.

\section{Dietary assessment}

Participants' diet was considered to assess the construct validity of the NKTS and its associations with adolescent food intakes. In the HELENA study, dietary intake data were collected using the validated HELENA-DIAT software by two non-consecutive computerized $24 \mathrm{~h}$ recalls, including weekdays and Sundays. The second assessment was performed within two weeks after the first one ${ }^{(13)}$. Adolescents autonomously selected all the foods and beverages consumed from a food list that included six 'meal occasions'. Some questions were presented to respondents to help them remember what they ate the day before. Some slides were presented for each food selected detailing the portion sizes ${ }^{(13,14)}$. In addition, a selfadministered FFQ was applied. This questionnaire included fifteen items and seven weekly response categories ranging from 'never' to 'more than once a day, every day' ${ }^{\text {(13) }}$. Both dietary assessment methods were administered during school time with the assistance of trained nutritionists.

Data from $24 \mathrm{~h}$ recalls regarding foods and beverages were expressed as grams and millilitres per day, respectively, and organized into food groups. Furthermore, to remove the effect of day-to-day variability and random error in the $24 \mathrm{~h}$ recalls, the individual usual food intake from these recalls was estimated using the multiple source method. This statistical modelling technique considers information from the FFQ as a covariate of the two $24 \mathrm{~h}$ recalls ${ }^{(15)}$.

In the present study, the foods groups that emerged from the $24 \mathrm{~h}$ recalls were categorized into fifteen food groups according to their similarity in nutritional content and health-related characteristics. These categories are: (i) vegetables (excluding potatoes); (ii) fruits; (iii) vegetables (excluding potatoes) and fruits; (iv) sweets (including carbonated/soft/isotonic drinks, cakes, biscuits, chocolate 
and other sugar products); (v) cereals (including potatoes); (vi) nuts and seeds; (vii) vegetable oils; (viii) olives and avocado; (ix) alcohol; (x) dairy products (including milk and yoghurt); (xi) pulses; (xii) meats and eggs; (xiii) fish; (xiv) savoury snacks (including chips, salty biscuits, crackers, and popcorn); and (xv) water.

\section{Anthropometric measurements}

Weight was measured with an electronic scale (model SECA 861) with $0 \cdot 1 \mathrm{~kg}$ precision. Height was measured with participants barefoot and head in the Frankfort plane using a telescopic height-measuring instrument (model SECA 225) with $0 \cdot 1 \mathrm{~cm}$ precision and range from 70 to $200 \mathrm{~cm}$. Both measurements were taken at the same time by trained staff ${ }^{(16)}$.

BMI $\left(\mathrm{kg} / \mathrm{m}^{2}\right)$ was calculated as weight (in kilograms) divided by the square of height (in metres). The values of BMI were categorized into 'thinness', 'normal weight', 'overweight' and 'obesity' considering the reference values for BMI in adults $(17,18)$.

\section{Biochemical analyses}

Blood biomarker concentrations were considered to assess the construct validity of the NKTS and its associations with adolescent nutrition. In the HELENA study, the blood collection was performed after a $10 \mathrm{~h}$ overnight fast following a standardized protocol and up to two weeks apart from the NKT. A handling and transportation system was developed to guarantee the quality assurance and stability of blood samples ${ }^{(19)}$.

Out of all the biomarkers analysed in the HELENA study, vitamin C, $\beta$-carotene, $n$-3 fatty acids, cobalamin and folate presented strong correlations with nutrient intakes and were included in the present analyses. Blood concentration of vitamin C, $\beta$-carotene and $n$ - 3 fatty acids presented a strong correlation with fruits, vegetables and $n$-3 fatty acids, respectively ${ }^{(13)}$. Trans-fatty acids were also considered due to their association with nutrition- and obesity-related diseases ${ }^{(20)}$. Folate (plasma and erythrocyte) and cobalamin were measured by a competitive immunoassay (Immulite 2000; DPC Biermann GmbH, Bad Nauheim, Germany). $\beta$-Carotene and vitamin $C$ were analysed by HPLC (Sykam, Gilching, Germany) using UV detection (UV-Vi 205; Merck, Darmstadt, Germany). Fatty acid concentrations were determined by capillary GC (model 3900) after extraction performed by thin-layer chromatography. The total amount of fatty acids was obtained from the percentage area by integrating the area under the peak and dividing it by the total area for all fatty acids. The labels $n-3$ and trans include all these types of fatty acids identified during blood analyses. Holotranscobalamin was measured by a microparticle enzyme immunoassay (Active $\mathrm{B}_{12}$; Axis-Shield Limited, Dundee, UK) with the use of an AxSYM analyser (Abbott Diagnostics, Abbott Park, IL, USA) ${ }^{(13,19)}$.
To measure plasma folate, cobalamin and vitamin C (previously stabilized with metaphosphoric acid) the blood sample was collected in heparinized tubes, transported on dry ice and centrifuged within $30 \mathrm{~min}$. The serum sample for fatty acid determination was also centrifuged within $30 \mathrm{~min}$, stored at $-80^{\circ} \mathrm{C}$ and transported on dry ice as soon as possible. The serum sample for $\beta$-carotene was stabilized with a synthetic antioxidant. For erythrocyte folate analysis, the whole blood was diluted in prepared ascorbic acid and incubated in the dark before storing it at $-80^{\circ} \mathrm{C}$. To measure holo-transcobalamin, blood was collected in evacuated tubes without anticoagulant and then the aliquot was stored at $-80^{\circ} \mathrm{C}$ until its transport to the laboratory ${ }^{(13,19)}$.

\section{Nutritional knowledge assessment}

Nutritional knowledge was evaluated with the NKT, a validated questionnaire designed by a nutritional psychologist $^{(21)}$. The NKT contains twenty-three multiplechoice items encompassing concepts related to energy intake and metabolism, nutrient contents, sweeteners and oral health, food knowledge, and special terms and definitions. This test was designed for those who did not receive any nutritional education programme in the past. Each item offers four possible answers, including a 'don't know' category, but only one answer category is correct. Furthermore, the NKT comprises common misconceptions as well as easy items in order to motivate individuals to answer the test. In the HELENA study, the NKT was completed by students during class with the supervision of the research team ${ }^{(3,21,22)}$.

\section{Statistical analyses}

The dimensionality of the NKT was analysed by factor analysis with estimation using principal component analysis and Varimax orthogonal rotation. The use of a onedimensional model was acceptable if the first principal component explained $20 \%$ or more of the total variance $^{(23)}$.

The biserial coefficient was calculated for each item to indicate the correlation behind the correct answer to items and the level of nutritional knowledge. Items with a negative biserial coefficient were excluded from the analyses $^{(24)}$.

A one-dimensional logistic model of the three parameters was used to calculate the probability of a person to correctly answer an item, with a given nutritional knowledge level. This model is represented by the following equation $^{(24)}$ :

$$
P\left(U_{i j}=1 \mid \theta_{j}\right)=c_{i}+\left(1-c_{i}\right) \frac{1}{1+\mathrm{e}^{-D a_{i}\left(\theta_{j}-b_{i}\right)}},
$$

where $\theta_{j}$ is the IRT score of a person $j ; P\left(U_{i j}=1 \mid \theta_{j}\right)$ is the probability of a person $j$ with an ability $\theta_{j}$ to correctly answer an item $i ; D$ is a scale constant factor and equal to 
$1.7 ; c_{i}$ is the guessing parameter of item $i ; a_{i}$ is the discrimination parameter of item $i$; and $b_{i}$ is the difficulty parameter of item $i$.

The discrimination parameter $a_{i}$ indicates the quality of an item to discriminate people with different levels of nutritional knowledge. Items with higher values on this parameter present better discrimination. The difficulty parameter $b_{i}$ identifies the point on the scale at which the probability to answer correctly is higher than or equal to $\left(1+c_{i}\right) / 2^{(24)}$. Finally, the guessing parameter $c_{i}$ indicates the probability that someone gives the correct answer by chance, with value equal to or above 0 and equal to or below $1\left(0 \leq c_{i} \leq 1\right)^{(25)}$.

All parameters $\left(a_{i}, b_{i}\right.$ and $\left.c_{i}\right)$ were estimated by marginal maximum likelihood with a maximum number of twenty quadrature points, fifty EM cycles, ten Newton interactions and convergence criterion equal to $0 \cdot 01$. These parameters estimates were analysed with the corresponding SE (see online supplementary material, Supplemental Table 1). Items with discrimination parameter $a_{i}$ value below or equal to 0.70 were considered as having less discrimination and were excluded from further analyses. The estimation of the parameters mentioned above was repeated only for items with adequate discrimination. To control the lack of identification of the model, $b_{i}$ and IRT scores were estimated on a scale with mean equal to 0 and SD equal to 1 by the expected $a$ posteriori method ${ }^{(25)}$. Empirical reliability, a precision measure analogous to Cronbach's $\alpha$, was calculated to analyse the reliability of the NKTS. Besides the empirical reliability, the test information curve was analysed to identify the accuracy of the measurement along the NKTS.

The probability of the correct answer for each item was calculated across IRT scores. The item was located at the level of the correct answer probability when it was higher than or equal to $\left(1+c_{i}\right) / 2$. Some levels were grouped into the same range of nutritional knowledge according to the technical similarity between items. This step was performed by two nutritionists (T.S.S.S. and C.J.) using Microsoft ${ }^{\circledR}$ Excel version 2010.

To analyse the associations between IRT scores and demographic, socio-economic and health characteristics, Kolmogorov-Smirnov tests were carried out to test the normality in the distribution of the studied variables and guide further analyses. The $\chi^{2}$ test was performed to evaluate differences between demographic, socio-economic and BMI categories by NKTS levels. Mixed-model linear regression analyses were performed to assess the construct validity of NKTS and its associations with nutritional knowledge, food group consumption and biomarkers. The IRT scores were included as the independent variable. To explore these associations, analyses were carried out separately for the NKTS (considering only items with adequate IRT parameters) and for all items in the original NKT (calculating all IRT scores although there were items with inadequate IRT parameters). Food groups and biomarker variables were included separately in the model with the outcome variable, and centre was included as the random intercept. Age, gender, maternal education, FAS index, BMI and energy intake were entered as covariates in the model. Statistical significance was set at $P<0.05$.

Analyses were performed using the statistical software package Stata version $14^{(26)}$ and the software BILOG-MG version $3^{(27)}$.

\section{Results}

As suggested by the factor analysis, the first principal component explained $46 \%$ of the total variance (see online supplementary material, Supplemental Fig. 1). None of the items presented a negative biserial coefficient (Supplemental Table 1). Twelve items presented low discrimination of nutritional knowledge and were excluded from further analyses. Biserial coefficient and IRT parameters $\left(a_{i}, b_{i}\right.$ and $\left.c_{i}\right)$ of the remaining items are described in Table 1.

The estimation of the IRT parameters was completed with eleven EM cycles and three Newton interactions. Along the nutritional knowledge continuum, the item 'A breakfast merely consisting of bread, jam and butter does not contain enough...?' presented the lowest discrimination parameter $\left(a_{i}=0.88\right)$. On the other hand, the item 'The ingredients list found on food items may contain a number of different terms for sugar. Which row lists three terms for special types of sugar?' had the highest discrimination parameter $\left(a_{i}=1.65\right)$.

The item 'Marcel has been playing with a ball all afternoon. During this time Kevin has been sitting at home watching television. Which of the following statements is most applicable?' had the lowest difficulty parameter $\left(b_{i}=-2 \cdot 13\right)$, suggesting that energy expenditure is a relatively easy concept for the adolescents. The item "Which row lists three terms for energy-free sweeteners?' presented the highest difficulty parameter $\left(b_{i}=1.50\right)$, suggesting that advanced nutritional terms were the most difficult ones to be identified by the HELENA adolescents.

All items presented low values of the guessing parameter $c_{i}$, with values ranging from 0.01 to 0.09 . In the studied sample, NKTS scores varied from $-0 \cdot 83$ to $0 \cdot 83$. NKTS showed a good empirical reliability (0.69) and presented more accurate information between IRT scores, ranging from $-1 \cdot 0$ to $1 \cdot 0$ (Fig. 2).

Results from the mixed-model linear regression analysis with food group consumption as the outcome variables and NKTS scores as the independent variable are shown in Table 2. Nutritional knowledge was positively associated with consumption of fruits, cereals, dairy products, pulses, meats and eggs, and fish, and was negatively associated with intake of olives and avocado, alcohol and savoury snacks. 
Table 1 Biserial coefficient and discrimination, difficulty and guessing parameters, with their SE, from the Item Response Theory analysis of the Nutritional Knowledge Scale

\begin{tabular}{|c|c|c|c|c|c|c|c|c|}
\hline Item & Correct answer & Biserial & $a_{i} \dagger$ & SE & $b_{i} \ddagger$ & SE & $c / \S$ & SE \\
\hline $\begin{array}{l}\text { Marcel has been playing with a ball all afternoon. During this } \\
\text { time Kevin has been sitting at home watching television. } \\
\text { Which of the following statements is most applicable? }\end{array}$ & $\begin{array}{l}\text { Marcel burns more energy than } \\
\text { Kevin }\end{array}$ & 0.44 & 1.22 & 0.09 & $-2 \cdot 13$ & 0.13 & 0.02 & 0.03 \\
\hline $\begin{array}{l}\text { Which row lists three dishes that have all been prepared } \\
\text { using very little fat? }\end{array}$ & $\begin{array}{l}\text { Boiled egg, boiled potatoes, } \\
\text { steamed fish }\end{array}$ & 0.37 & 0.91 & 0.06 & $-1 \cdot 13$ & 0.09 & 0.02 & 0.02 \\
\hline Which substance is good for your teeth? & Fluoride & 0.39 & 0.91 & 0.07 & -0.66 & 0.10 & 0.02 & 0.03 \\
\hline Bread, cake, pasta, potatoes and rice contain mainly...? & Carbohydrates & 0.45 & $1 \cdot 17$ & 0.13 & $-0 \cdot 19$ & 0.16 & 0.09 & 0.07 \\
\hline $\begin{array}{l}\text { Dietitians use the American term 'junk food' to describe } \\
\text { certain foods. What do they mean by this? }\end{array}$ & $\begin{array}{l}\text { Foods that contain a lot of } \\
\text { energy but are of very little } \\
\text { nutritional value }\end{array}$ & 0.41 & 0.98 & 0.07 & $-0 \cdot 18$ & 0.07 & 0.02 & 0.02 \\
\hline $\begin{array}{l}\text { What effect does the fibre contained in our food have on the } \\
\text { human body? }\end{array}$ & $\begin{array}{l}\text { It stimulates the process of } \\
\text { digestion }\end{array}$ & 0.51 & 1.32 & 0.09 & 0.02 & 0.06 & 0.02 & 0.02 \\
\hline $\begin{array}{l}\text { The ingredients list found on food items may contain a } \\
\text { number of different terms for sugar. Which row lists three } \\
\text { terms for special types of sugar? }\end{array}$ & Dextrose, fructose, maltose & 0.56 & 1.65 & 0.11 & $0 \cdot 19$ & 0.04 & 0.01 & 0.01 \\
\hline $\begin{array}{l}\text { A breakfast merely consisting of bread, jam and butter does } \\
\text { not contain enough...? }\end{array}$ & Protein & 0.38 & 0.88 & 0.08 & 0.35 & 0.12 & 0.03 & 0.04 \\
\hline Which row lists three foods that contain a lot of vitamin $C$ ? & Peppers, cabbage, citrus fruit & 0.40 & 0.98 & $0 \cdot 10$ & 0.48 & 0.11 & 0.04 & 0.04 \\
\hline What is the other commonly used term for energy? & Joule & 0.41 & 1.08 & 0.11 & 0.96 & 0.08 & 0.03 & 0.03 \\
\hline Which row lists three terms for energy-free sweeteners? & $\begin{array}{l}\text { Aspartame, saccharin, } \\
\text { cyclamate }\end{array}$ & 0.45 & $1 \cdot 28$ & 0.14 & 1.50 & 0.08 & 0.01 & 0.01 \\
\hline
\end{tabular}

†Discrimination parameter.

¥Difficulty parameter.

$\S$ Guessing parameter.

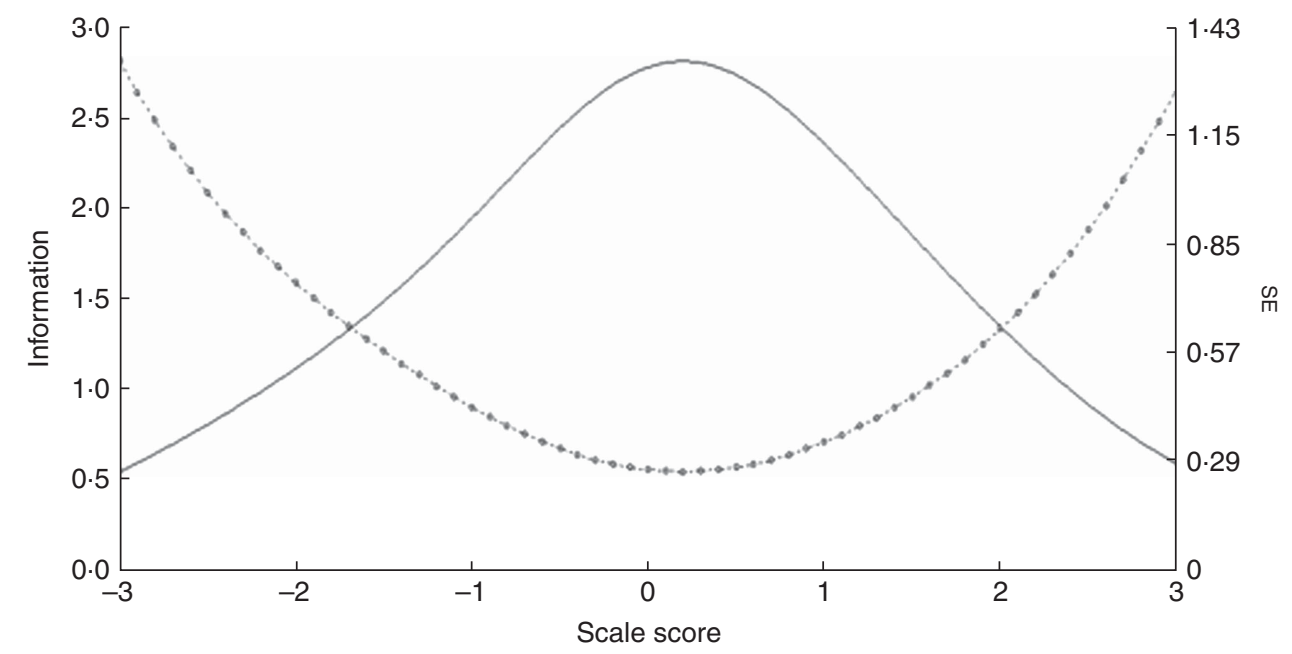

Fig. 2 Nutritional Knowledge Scale information curve ( $n$ 3215): _—_, test information curve; ----, standard error curve

Results from the mixed-model linear regression analysis with the concentration of biomarkers as the outcome variables and NKTS scores as the independent variable are presented in Table 3. Nutritional knowledge was positively associated with blood concentrations of vitamin C, $\beta$-carotene, $n$-3 fatty acids, holo-transcobalamin, cobalamin and folate. Additionally, both mixed-model linear regression analyses presented similar results when IRT scores were calculated for all items of the original NKT that presented low discrimination of nutritional knowledge (see online supplementary material, Supplemental Tables 2 and 3) and when the number of cigarettes consumed was included as a covariate (data not shown).
NKTS scores were categorized into three different levels to facilitate their interpretation: basic, adequate and advanced. The basic level (IRT scores below -0.5) included knowledge about energy expenditure and ingredients from recipes. This level comprised the items: 'Marcel has been playing with a ball all afternoon. During this time Kevin has been sitting at home watching television. Which of the following statements is most applicable?' and 'Which row lists three dishes that have all been prepared using very little fat?' The adequate level (IRT scores from -0.5 to 1.0) included knowledge about nutrients from foods and their role in health. This level presented the following items: 'Which substance is good for your teeth?'; 'Bread, 
Table 2 Mixed-model analyses between intakes of food groups $(\mathrm{g} / \mathrm{d})$ and Item Response Theory scores from the Nutritional Knowledge Scale, adjusted for age, gender, maternal education, Family Affluence Scale index and energy intake, among European adolescents aged 12.5-17.5 years ( $n$ 1623), HELENA (Healthy Lifestyle in Europe by Nutrition in Adolescence) study

\begin{tabular}{|c|c|c|c|}
\hline Food group & $\beta \dagger$ & $95 \% \mathrm{Cl}$ & $P$ value \\
\hline Vegetables & -33.03 & $-78 \cdot 88,12 \cdot 81$ & 0.16 \\
\hline Fruits & 109.75 & $30.53,188.96$ & $0.01^{*}$ \\
\hline Fruits and vegetables & 83.58 & $-9 \cdot 17,176.33$ & 0.08 \\
\hline Sweets & -98.80 & $-338.44,140.83$ & 0.42 \\
\hline Cereals & 114.42 & $51.77,177.06$ & $<0.001^{*}$ \\
\hline Nuts and seeds & -4.25 & $-11.41,2.91$ & 0.24 \\
\hline Olives and avocado & $-6 \cdot 19$ & $-11.60,-0.79$ & $0.02^{*}$ \\
\hline Vegetable oils & $-2 \cdot 21$ & $-9 \cdot 10,4 \cdot 67$ & 0.53 \\
\hline Alcohol & -160.41 & $-237.06,-83.77$ & $<0.001^{*}$ \\
\hline Dairy products & 401.06 & $233.18,568.93$ & $<0.001^{*}$ \\
\hline Pulses & $32 \cdot 29$ & $9.88,54.69$ & $<0.01^{*}$ \\
\hline Water & 293.82 & $-122.91,710.55$ & 0.17 \\
\hline Fish & 39.91 & $21.46,58.36$ & $<0.001^{\star}$ \\
\hline Meats and eggs & $150 \cdot 28$ & $96 \cdot 12,204.45$ & $<0.001^{*}$ \\
\hline Savoury snacks & -14.85 & $-27 \cdot 40,-2 \cdot 30$ & $0.02^{*}$ \\
\hline
\end{tabular}

Centre was used as the random intercept.

*Significant $P$ values.

†Fixed-effects estimates.

Table 3 Mixed-model analyses between concentrations of biomarkers and Item Response Theory scores from the Nutritional Knowledge Scale, adjusted for age, gender, maternal education, Family Affluence Scale index, BMl and energy intake, among European adolescents aged 12.5-17.5 years ( $n 609$ ), HELENA (Healthy Lifestyle in Europe by Nutrition in Adolescence) study

\begin{tabular}{|c|c|c|c|}
\hline Biomarker & $\beta \dagger$ & $95 \% \mathrm{Cl}$ & $P$ value \\
\hline Vitamin C (mg/l) & $10 \cdot 90$ & $5 \cdot 68,16 \cdot 12$ & $<0.001^{*}$ \\
\hline$\beta$-Carotene $(\mathrm{ng} / \mathrm{ml})$ & 476.05 & $179.56,772.54$ & $0.002^{*}$ \\
\hline$n-3$ Fatty acids (\%) & 5.09 & $2.89,7.29$ & $<0.001^{*}$ \\
\hline$n-3$ Fatty acids $(\mu \mathrm{mol} / \mathrm{l})$ & $155 \cdot 21$ & $71 \cdot 34,239.08$ & $<0.001^{*}$ \\
\hline Holo-transcobalamin (pmol/l) & $66 \cdot 22$ & $11.23,121.22$ & $0.02^{*}$ \\
\hline Cobalamin (pmol/l) & 539.86 & $335 \cdot 23,744 \cdot 49$ & $<0.001^{*}$ \\
\hline Plasma folate $(\mathrm{nmol} / \mathrm{l})$ & $42 \cdot 24$ & $25 \cdot 37,59 \cdot 11$ & $<0.001^{*}$ \\
\hline Erythrocyte folate $(\mathrm{nmol} / \mathrm{l})$ & 1253.45 & $648.63,1858.27$ & $<0.001^{*}$ \\
\hline Trans-fatty acids $(\mu \mathrm{mol} / \mathrm{l})$ & $1 \cdot 20$ & $-0.01,2.41$ & 0.05 \\
\hline
\end{tabular}

Centre was used as the random intercept.

*Significant $P$ values.

†Fixed-effects estimates.

cake, pasta, potatoes and rice contain mainly...?'; 'Dietitians use the American term "junk food" to describe certain foods. What do they mean by this?'; 'What effects does the fibre contained in our food have on the human body?'; 'The ingredients list found on food items may contain a number of different terms for sugar. Which row lists three terms for special types of sugar?'; 'A breakfast merely consisting of bread, jam and butter does not contain enough...?'; and 'Which row lists three foods that contain a lot of vitamin C?' The advanced level (IRT scores above or equal to 1.0) included knowledge about basic and advanced nutritional terms. This last level contained the items: "What is the other commonly used term for energy?' and 'Which row lists three terms for energy-free sweeteners?' Therefore, a person who shows a nutritional knowledge level below -0.5 would have knowledge on energy expenditure and ingredients from recipes. On the other hand, a person with a nutritional knowledge level above or equal to 1 would present advanced nutritional knowledge, such as knowledge of energy expenditure, nutrients from foods and their role in health, ingredients from recipes and advanced nutritional terms.

The demographic and socio-economic characteristics of participants are described in Table 4. Girls, older adolescents, those whose mothers had a university education and those with a high FAS index presented higher nutritional knowledge.

\section{Discussion}

When evaluating if the NKT assessed nutritional knowledge, factor analysis indicated that the first principal component explained $46 \%$ of the total variance. Therefore, an one-dimensional logistic model of three parameters was applied to develop the $\operatorname{NKTS}^{(23,24)}$.

The original NKT calculated only the percentage of correct answers ${ }^{(3)}$; in this way, adolescents with different 
Table 4 Demographic and socio-economic characteristics, by Nutritional Knowledge Scale level, of European adolescents aged 12.5-17.5 years ( $n$ 3215), HELENA (Healthy Lifestyle in Europe by Nutrition in Adolescence) study

\begin{tabular}{|c|c|c|c|c|c|c|c|c|c|}
\hline & \multirow{2}{*}{\multicolumn{2}{|c|}{ Total sample }} & \multicolumn{6}{|c|}{ Nutritional Knowledge Scale level } & \multirow[b]{3}{*}{$P$ value- } \\
\hline & & & \multicolumn{2}{|c|}{$\begin{array}{c}\text { Basic } \\
(n 376 ; 11.7 \%)\end{array}$} & \multicolumn{2}{|c|}{$\begin{array}{c}\text { Adequate } \\
\text { (n 1912; 59.5\%) }\end{array}$} & \multicolumn{2}{|c|}{$\begin{array}{c}\text { Advanced } \\
\text { (n 927; } 28.8 \%)\end{array}$} & \\
\hline & $n$ & $\%$ & $n$ & $\%$ & $n$ & $\%$ & $n$ & $\%$ & \\
\hline Age (years) & & & & & & & & & $<0.001^{*}$ \\
\hline $12.5-13.99$ & 1022 & 31.8 & 161 & $42 \cdot 8$ & 656 & 34.3 & 205 & $22 \cdot 1$ & \\
\hline $14-14.99$ & 795 & 24.7 & 99 & $26 \cdot 3$ & 482 & $25 \cdot 2$ & 214 & $23 \cdot 1$ & \\
\hline $15-15.99$ & 783 & 24.4 & 65 & 17.3 & 457 & 23.9 & 261 & 28.2 & \\
\hline $16-17.49$ & 615 & $19 \cdot 1$ & 51 & $13 \cdot 6$ & 317 & $16 \cdot 6$ & 247 & $26 \cdot 6$ & \\
\hline Gender & & & & & & & & & $0.04^{*}$ \\
\hline Male & 1526 & 47.5 & 183 & $48 \cdot 7$ & 936 & $49 \cdot 0$ & 407 & 43.9 & \\
\hline Female & 1689 & 52.5 & 193 & $51 \cdot 3$ & 976 & $51 \cdot 0$ & 520 & $56 \cdot 1$ & \\
\hline FAS index ( $n$ 3044) & & & & & & & & & $<0.001^{*}$ \\
\hline Low & 1397 & $45 \cdot 9$ & 198 & $57 \cdot 7$ & 852 & $47 \cdot 1$ & 347 & 38.8 & \\
\hline High & 1647 & $54 \cdot 1$ & 145 & $42 \cdot 3$ & 955 & 52.9 & 547 & $61 \cdot 2$ & \\
\hline Maternal education ( $n$ 3072) & & & & & & & & & $<0.001^{*}$ \\
\hline Primary & 252 & $8 \cdot 2$ & 43 & 12.5 & 168 & $9 \cdot 2$ & 41 & 4.6 & \\
\hline Lower secondary & 819 & $26 \cdot 7$ & 104 & $30 \cdot 2$ & 533 & $29 \cdot 0$ & 182 & 20.4 & \\
\hline Higher secondary & 966 & 31.4 & 100 & $29 \cdot 1$ & 568 & 31.0 & 298 & 33.4 & \\
\hline University degree & 1035 & 33.7 & 97 & $28 \cdot 2$ & 566 & $30 \cdot 8$ & 372 & 41.7 & \\
\hline Centre & & & & & & & & & $<0.001^{*}$ \\
\hline Athens & 272 & 8.5 & 80 & $21 \cdot 3$ & 149 & $7 \cdot 8$ & 43 & 4.6 & \\
\hline Dortmund & 422 & 13.1 & 55 & 14.6 & 290 & $15 \cdot 2$ & 77 & 8.3 & \\
\hline Ghent & 322 & $10 \cdot 0$ & 28 & $7 \cdot 4$ & 196 & $10 \cdot 3$ & 98 & $10 \cdot 6$ & \\
\hline Heraklion & 245 & $7 \cdot 6$ & 59 & $15 \cdot 7$ & 154 & $8 \cdot 1$ & 32 & 3.5 & \\
\hline Lille & 243 & 7.6 & 35 & $9 \cdot 3$ & 189 & 9.9 & 19 & $2 \cdot 0$ & \\
\hline Pécs & 376 & 11.7 & 29 & $7 \cdot 7$ & 199 & 10.4 & 148) & $16 \cdot 0$ & \\
\hline Rome & 293 & $9 \cdot 1$ & 21 & $5 \cdot 6$ & 195 & $10 \cdot 2$ & 77 & 8.3 & \\
\hline Stockholm & 292 & $9 \cdot 1$ & 36 & $9 \cdot 6$ & 173 & $9 \cdot 0$ & 83 & $9 \cdot 0$ & \\
\hline Vienna & 383 & 11.9 & 24 & $6 \cdot 4$ & 174 & $9 \cdot 1$ & 185 & $20 \cdot 0$ & \\
\hline Zaragoza & 367 & 11.4 & 9 & 2.4 & 193 & $10 \cdot 1$ & 165 & $17 \cdot 8$ & \\
\hline BMI & & & & & & & & & $0.01^{*}$ \\
\hline Thinness & 193 & $6 \cdot 0$ & 28 & 7.4 & 109 & 5.7 & 56 & $6 \cdot 0$ & \\
\hline Normal weight & 2280 & 70.9 & 248 & $66 \cdot 0$ & 1346 & $70 \cdot 4$ & 686 & 74.0 & \\
\hline Overweight & 561 & $17 \cdot 4$ & 67 & 17.8 & 347 & $18 \cdot 1$ & 147 & $15 \cdot 9$ & \\
\hline Obesity & 181 & $5 \cdot 6$ & 33 & 8.8 & 110 & $5 \cdot 8$ & 38 & $4 \cdot 1$ & \\
\hline
\end{tabular}

FAS, Family Affluence Scale.

* Significant $P$ values.

†Likelihood ratio $P$ value.

nutritional knowledge levels could get the same score ${ }^{(28)}$. The IRT analysis complements the classical approach as it calculates the scores and the pattern of correct answers by comparing the difficulty of each item ${ }^{(29)}$.

Twelve items from the original NKT presented low discrimination of nutritional knowledge and thus were excluded. The NKTS provides a high-quality tool to assess nutritional knowledge, as it includes items with the best discrimination of nutritional knowledge. In addition, results from the mixed-model linear regression analyses presented similar associations between food group consumption and nutritional biomarkers in two different approaches: when considering the NKTS (IRT scores calculated from eleven items) or the original NKT (IRT scores calculated from all twenty-three items). One of the benefits of using a lower number of items is the accuracy of participants' responses, as large periods for questionnaire completion tend to increase fatigue and boredom ${ }^{(30)}$. Therefore, the reduction in the number of items on the questionnaire may improve the quality of answers and decrease the time of completion. This aspect is important for epidemiology researchers working with large sample sizes and assessing information in a short period of time.

Besides this refinement of the NKT, by selecting the items that best discriminated nutritional knowledge, all items maintained on the NKTS presented an adequate guessing parameter. If an item presents a high value concerning the guessing parameter, it should be excluded from the final questionnaire because guessing is influenced by a high probability of correct answers ${ }^{(24)}$. Therefore, adequate guessing parameters may confirm the precision of the NKTS. In this regard, the IRT analyses improved the quality of nutritional knowledge assessment.

In addition to the reduced number of items and greater accuracy of the measurement, the current analysis confirmed the construct validity of the NKTS. The mixedmodel linear regression analysis confirmed that nutritional knowledge is positively associated with the consumption of healthy foods and an appropriate profile of nutritional biomarkers. The NKTS scores were positively associated 
with intakes of fruits, cereals, dairy products, pulses, meat and eggs, and fish, as well as with blood concentrations of vitamin C, $\beta$-carotene, $n$-3 fatty acids, holo-transcobalamin, cobalamin and folate; on the other hand, nutritional knowledge was negatively associated with consumption of olives and avocado, alcohol and savoury snacks. A recent study on Japanese children and their guardians also observed a positive association between nutritional knowledge and consumption of healthy foods ${ }^{(31)}$. Among adolescents, some studies have demonstrated that nutritional knowledge is related to a higher overall variety of food $^{(32,33)}$, higher serum retinol and lower intakes of total fat and added sugars ${ }^{(33)}$. Finally, considering the challenges to obtain accurate food intakes, the positive associations between NKTS scores and nutritional biomarkers improved the results from the mixed-model linear regression analysis with food groups' consumption as the outcome variable. Despite the non-expected negative association with intakes of olives and avocado $(P<0.05)$, our results highlight the importance of acquiring nutritional knowledge as a strategy to improve dietary habits among adolescents.

After calculating IRT scores, the next step was to describe the nutritional knowledge in each NKTS level. Based on this description, it may be possible to better assess what adolescents know about nutrition and with which items they may have more difficulty ${ }^{(29)}$. The basic level includes knowledge about energy expenditure and ingredients from recipes. Individuals who are classified on this level would correctly answer to the item with the lowest difficulty level: 'Marcel has been playing with a ball all afternoon. During this time Kevin has been sitting at home watching television. Which of the following statements is most applicable?' $\left(b_{i}=-2 \cdot 13\right)$. The item 'Which row lists three terms for energy-free sweeteners?' presented the highest difficulty parameter $\left(b_{i}=1 \cdot 50\right)$, suggesting that advanced nutritional terms were the most difficult to be identified by adolescents of the HELENA study. This item would probably be answered correctly by those adolescents on the advanced level and with NKTS scores higher than 1.50. Therefore, the identification of the NKTS levels in the studied individuals may improve the strategic direction of scientific interventions and policy making.

This description makes it possible to determine the proportion of individuals on each NKT level. In this regard, adolescents from Heraklion and Lille presented the lowest NKTS scores. In agreement with a previous study that evaluated the $\mathrm{NKT}^{(3)}$, the scores from the NKTS significantly increased in adolescents who were older in age, female and whose mothers had more education. Moreover, the positive associations between NKTS scores and socio-economic level are consistent with previous research. A population-based study indicated a linear association between nutritional knowledge and socio-economic level in urban and rural regions in
Iraq ${ }^{(34)}$. Finally, in the current study, NKTS scores were negatively associated with BMI among thin and obese adolescents. However, the relationship between nutritional knowledge and nutritional status is not well known in the literature. The previous study evaluating the $\mathrm{NKT}^{(3)}$ and other studies have demonstrated no significant associations in adolescents and adults ${ }^{(35,36)}$. However, it is important to mention that these studies used classical test theory and, therefore, their results may not be accurate.

The test information curve indicated that the NKTS is more precise within medium scores. For this reason, it is necessary to include new items measuring other levels of knowledge. In future studies, the item parameters could be maintained and new items included in the NKTS. This could be possible using computerized adaptive testing which allows calculating more precise estimates and creating a flexible questionnaire making it less burdensome for respondents ${ }^{(37)}$.

The current analysis aimed to evaluate the NKT based on the ITR analysis and assess the construct validity of the developed NKTS and its associations with food consumption and nutritional biomarkers in adolescents. The factor analysis confirmed that the NKT assessed nutritional knowledge. However, if we were evaluating nutritional knowledge in other populations, the classical approach would not be applicable by using the original NKT. The IRT analysis complemented the classical test approach, identifying those items with better discrimination and the location of each item on the continuum. Additionally, IRT analysis added the characterization of each nutritional knowledge level. Results from the mixedmodel linear regression analysis confirmed the validity of the NKTS, endorsing its use in epidemiological studies. Furthermore, due to the invariance in the estimation of the IRT parameters, the NKTS can be applied to other populations such as adolescents and adults from different countries $^{(28)}$. When analysing NKTS scores in other populations it will be necessary that participants answer some or all of the eleven items. Moreover, to calculate these scores, it is important to consider the IRT parameters calculated in the current study. In addition, IRT scores can be applied to further studies in continuous or categorical forms.

\section{Limitations and strengths}

One limitation of the present study concerns the crosssectional design which does not allow pointing out causal inferences. Besides, the missing values reduced the sample size for the IRT analysis, but it was large enough to estimate IRT parameters and detect associations between IRT scores and both food intakes and nutritional biomarkers. The HELENA study did not assess information on supplement intake that may affect the results from the mixed-model linear regression analyses with the 
concentration of biomarkers as the dependent variable. The dietary assessment was based on self-reporting which may affect the precision of the information, although the HELENA-DIAT software includes some questions to help adolescents remember what they ate the day before. However, the study has some strengths; the stratified nature of the sampling increased the precision of the data that may differ across strata. The standard procedures used to collect all measurements throughout the cities and the analyses were controlled for several confounding factors. Furthermore, the development of the NKTS was based on statistical analyses that solve the limitations of the classical test approach previously described. In this regard, the use of the NKTS may generate new and promising research partnerships, and after its standardization, researchers can apply this new method to assess nutritional knowledge in different studies and populations.

\section{Conclusions}

The IRT analysis selected those items from the NKT which presented better nutritional knowledge discrimination. The NKTS provides a more precise assessment of the latent trait with a short and high-quality pool of items. The mixed-model linear regression analysis confirmed the validity of the NKTS, indicating positive associations between the NKTS and healthy food consumption as well as nutritional biomarkers. The NKTS is proposed as a new instrument to assess nutritional knowledge in Europe and other parts of the world.

\section{Acknowledgements}

Financial support: The HELENA study took place with the financial support of the European Community Sixth RTD Framework Programme (contract FOOD-CT-2005007034). The present study was supported by a grant from Exterior Sandwich Doctorate Program for the Coordination of Improvement of Higher Level Personnel (CAPES, Coordenação de Aperfeiçoamento de Pessoal de Nível Superior) (grant number 88881.132853/2016-01). The European Community Sixth RTD Framework Programme and CAPES had no role in the design, analysis or writing of this article. Conflict of interest: None. Authorship: T.S.S.S. and C.J. analysed the data and wrote the first draft with contributions from L.A.M. All authors reviewed and commented on subsequent drafts of the manuscript. Ethics of buman subject participation: This study was conducted according to the guidelines laid down in the Declaration of Helsinki and all procedures involving human subjects were approved by the responsible institution in each of the participating countries. Written informed consent was obtained from all participants.

\section{Supplementary material}

To view supplementary material for this article, please visit https://doi.org/10.1017/\$1368980018003269

\section{References}

1. Robertson A, Tirado C, Lobstein T et al. (editors) (2004) Food and Health in Europe: A New Basis for Action. WHO Regional Publications, European Series no. 96. Copenhagen: WHO Regional Office for Europe.

2. Parmenter K \& Wardle J (2000) Evaluation and design of nutritional knowledge measures. J Nutr Educ 32, 269-277.

3. Sichert-Hellert W, Beghin L, De Henauw S et al. (2011) Nutritional knowledge in European adolescents: results from the HELENA (Healthy Lifestyle in Europe by Nutrition in Adolescence) study. Public Health Nutr 14, 2083-2091.

4. Dickson-Spillmann M, Siegrist M \& Keller C (2011) Development and validation of a short, consumer-oriented nutrition knowledge questionnaire. Appetite 56, 617-620.

5. Whati LH, Senekal M, Steyn NP et al. (2005) Development of a reliable and valid nutritional knowledge questionnaire for urban South African adolescents. Nutrition 21, 76-85.

6. Nguyen TH, Han HR, Kim MT et al. (2014) An introduction to Item Response Theory for patient-reported outcome measurement. Patient 7, 23-35.

7. Moreno LA, De Henauw S, Gonzalez-Gross M et al. (2008) Design and implementation of the Healthy Lifestyle in Europe by Nutritional in Adolescence Cross-Sectional Study. Int J Obes (Lond) 32, Suppl. 5, S4-S11.

8. Goldberg GR, Black AF, Jebb SA et al. (1991) Critical evaluation of energy intake data using fundamental principles of energy physiology: 1. Derivation of cut-off limits to identify under-recording. Eur J Clin Nutr 45, 569-581.

9. Black AF (2000) Critical evaluation of energy intake using the Goldberg cut-off for energy intake:basal metabolic rate. A practical guide to its calculation, use and limitations. Int $J$ Obes Relat Metab Disord 24, 1119-1130.

10. Beghin L, Castera M, Manios Y et al. (2009) Quality assurance of ethical issues and regulatory aspects relating to good clinical practices in the HELENA CrossSectional Study. Int J Obes (Lond) 32, Suppl. 5, S12-S18.

11. Iliescu C, Beghin L, Maes L et al. (2008) Socioeconomic questionnaire and clinical assessment in the HELENA Cross-Sectional Study: methodology. Int J Obes (Lond) 32, Suppl. 5, S19-S25.

12. Julian C, Mouratidou T, Vicente-Rodrigues G et al. (2017) Dietary sources and sociodemographic and lifestyle factors affecting vitamin D and calcium intakes in European adolescents: the Healthy Lifestyle in Europe by Nutrition in Adolescence (HELENA) Study. Public Health Nutr 20, 1593-1601.

13. Vandevijvere S, Geelen A, Gonzalez-Gross M et al. (2013) Evaluation of food and nutrient intake assessment using concentration biomarkers in European adolescents from the Healthy Lifestyle in Europe by Nutrition in Adolescence study. BrJ Nutr 109, 736-747.

14. Vereecken CA, Covents M, Sichert-Hellert W et al. (2008) Development and evaluation of a self-administered computerized $24 \mathrm{~h}$ dietary recall method for adolescents in Europe. Int J Obes (Lond) 32, Suppl. 5, S26-S34.

15. Currie C (editor) (1998) Health Behavior in School-aged Children: A WHO Cross-National Survey (HBC) Research Protocol for the 1997/1998 Survey. Edinburgh: University of Edinburgh, Research Unit in Health and Behavioural Change.

16. Nagy E, Vicente-Rodriguez G, Manios Y et al. (2008) Harmonization process and reliability assessment of anthropometric measurements in a multicenter study in adolescents. Int J Obes (Lond) 32, Suppl. 5, S58-S65. 
17. Cole TJ, Flegal KM, Nicholls D et al. (2007) Body mass index cut offs to define thinness in children and adolescents: international survey. BMJ 335, 194-202.

18. Cole TJ, Bellizzi MC, Flegal KM et al. (2000) Establishing a standard definition for child overweight and obesity worldwide: international survey. BMJ 320, 1240-1243.

19. González-Gross M, Breidenasel C, Gomez-Martinez S et al. (2008) Sampling and processing of fresh blood samples within a European multicenter nutritional study: evaluation of biomarker stability during transport and storage. Int J Obes (Lond) 32, Suppl. 5, S66-S75.

20. Wang Q, Inamura F, Lemaitre RN et al. (2014) Plasma phospholipid trans-fatty acids levels, cardiovascular diseases, and total mortality: the Cardiovascular Health Study. $J$ Am Heart Assoc 3, e000914.

21. Diehl JM (1999) Ernährungswissen von Kindern und Jugendlichen. Verbraucherdienst 44, 282-287.

22. Kersting M, Sichert-Hellert W, Vereecken CA et al. (2008) Food and nutrient intake, nutritional knowledge and dietrelated attitudes in European adolescents. Int J Obes (Lond) 32, Suppl. 5, S35-S41.

23. Reckase MD (1979) Unifactor latent trait models applied to multifactor tests: results and implications. J Educ Behav Stat 4, 207-230

24. Andrade DF, Tavares HR \& Valle RC (2000) Teoria de Resposta ao Item: Conceitos e Aplicações. In $4^{\circ}$ Simpósio Nacional de Probabilidade e Estatística - SINAPE 2000, Caxambu, Minas Gerais, Brazil. São Paulo: Associação Brasileira de Estatística.

25. Chernyshenko O, Stark S, Chan K et al. (2001) Fitting Item Response Theory models to two personality inventories: issues and insights. Multivariate Behav Res 36, 523-562.

26. StataCorp (2015) Stata Statistical Software: Release 14. College Station, TX: StataCorp LP.

27. Zimowski MF, Muraki E, Mislevy RJ et al. (2003) BILOG-MG 3. Chicago, IL: Scientific Software.

28. Wilson M, Allen DD \& Li JC (2006) Improving measurement in health education and health behavior research using item response modeling: comparison with the classical test theory approach. Health Educ Res 21, Suppl. 1, i19-i32.

29. Primi R (2004) Advances in scale interpretation with the application of Item Response Theory. Aval Psicol 3, 53-58.

30. Burns KEA, Duffett M, Kho ME et al. (2008) A guide for the design and conduct of self-administered surveys of clinicians. CMAJ 179, 245-252.

31. Asakura K, Todoriki K \& Sasaki S (2017) Relationship between nutrition knowledge and dietary intake among primary school children in Japan: combined effect of children's and their guardians' knowledge. J Epidemiol 27, 483-491.

32. Augustine LF, Vazir S, Rao SF et al. (2012) Psychometric validation of a knowledge questionnaire on micronutrients among adolescents and its relationship to micronutrient status of 15-19-year-old adolescent boys, Hyderabad, India. Public Health Nutr 15, 1182-1189.

33. Oldewage-Theron W, Egal A \& Moroka T (2014) Nutrition knowledge and dietary intake of adolescents in Cofimvaba, Eastern Cape, South Africa. Ecol Food Nutr 54, 138-156.

34. Heshmat R, Salehi F, Qorbani M et al. (2016) Economic inequality in nutritional knowledge, attitude and practice of Iranian households: the NUTRI-KAP study. Med J Islam Repub Iran 30, 915-922.

35. Brien GO \& Davies M (2005) Nutrition knowledge and body mass index. Health Educ Res 22, 571-575.

36. Meseri R, Mermer G, Ergin I et al. (2015) Evaluation of obesity prevalence and nutritional knowledge in adolescents in semi urban area of Turkey. Prog Nutr 17, 58-67.

37. Cella D, Riley W, Stone A et al. (2010) The patient-reported outcomes measurement information system (PROMIS) developed and tested its first wave of adult self-reported health outcome item banks: 2005-2008. J Clin Epidemiol 63, 1179-1194.

\section{Appendix}

\section{The HELENA Study Group}

Co-ordinator: Luis A. Moreno. Core Group members: Luis A. Moreno, Fréderic Gottrand, Stefaan De Henauw, Marcela González-Gross, Chantal Gilbert. Steering Committee: Anthony Kafatos (President), Luis A. Moreno, Christian Libersa, Stefaan De Henauw, Sara Castelló, Fréderic Gottrand, Mathilde Kersting, Michael Sjöstrom, Dénes Molnár, Marcela González-Gross, Jean Dallongeville, Chantal Gilbert, Gunnar Hall, Lea Maes, Luca Scalfi. Project Manager: Pilar Meléndez. Universidad de Zaragoza (Spain): Luis A. Moreno, Jesús Fleta, José A. Casajús, Gerardo Rodríguez, Concepción Tomás, María I. Mesana, Germán Vicente-Rodríguez, Adoración Villarroya, Carlos M. Gil, Ignacio Ara, Juan Fernández Alvira, Gloria Bueno, Aurora Lázaro, Olga Bueno, Juan F. León, Jesús $M^{a}$ Garagorri, Idoia Labayen, Iris Iglesia, Silvia Bel, Luis A. Gracia Marco, Theodora Mouratidou, Alba Santaliestra-Pasías, Iris Iglesia, Esther González-Gil, Pilar De Miguel-Etayo, Cristina Julian, Mary Miguel-Berges, Isabel Iguacel. Consejo Superior de Investigaciones Científicas (Spain): Ascensión Marcos, Julia Wärnberg, Esther Nova, Sonia Gómez, Ligia Esperanza Díaz, Javier Romeo, Ana Veses, Belén Zapatera, Tamara Pozo, David Martínez. Université de Lille 2 (France): Laurent Beghin, Christian Libersa, Frédéric Gottrand, Catalina Iliescu, Juliana Von Berlepsch. Research Institute of Child Nutrition Dortmund, Rheinische Friedrich-Wilhelms-Universität Bonn (Germany): Mathilde Kersting, Wolfgang Sichert-Hellert, Ellen Koeppen. Pécsi Tudományegyetem (University of Pécs) (Hungary): Dénes Molnar, Eva Erhardt, Katalin Csernus, Katalin Török, Szilvia Bokor, Mrs Angster, Enikö Nagy, Orsolya Kovács, Judit Répasi. University of Crete School of Medicine (Greece): Anthony Kafatos, Caroline Codrington, María Plada, Angeliki Papadaki, Katerina Sarri, Anna Viskadourou, Christos Hatzis, Michael Kiriakakis, George Tsibinos, Constantine Vardavas, Manolis Sbokos, Eva Protoyeraki, Maria Fasoulaki. Institut für Ernährungsund Lebensmittelwissenschaften - Ernährungphysiologie, Rheinische Friedrich Wilhelms Universität (Germany): Peter Stehle, Klaus Pietrzik, Marcela González-Gross, Christina Breidenassel, Andre Spinneker, Jasmin Al-Tahan, Miriam Segoviano, Anke Berchtold, Christine Bierschbach, Erika Blatzheim, Adelheid Schuch, Petra Pickert. University of Granada (Spain): Manuel J. Castillo, Ángel Gutiérrez, Francisco B. Ortega, Jonatan R. Ruiz, Enrique G. Artero, Vanesa España, David Jiménez-Pavón, Palma Chillón, Cristóbal Sánchez-Muñoz, Magdalena Cuenca. Instituto Nazionalen di Ricerca per gli Alimenti e la Nutrizione (Italy): Davide Arcella, Elena Azzini, Emma Barrison, Noemi Bevilacqua, Pasquale Buonocore, Giovina Catasta, Laura Censi, Donatella Ciarapica, Paola D'Acapito, Marika Ferrari, Myriam Galfo, Cinzia Le Donne, Catherine Leclercq, Giuseppe Maiani, Beatrice Mauro, Lorenza Mistura, Antonella Pasquali, Raffaela Piccinelli, 
Angela Polito, Romana Roccaldo, Raffaella Spada, Stefania Sette, Maria Zaccaria. University of Napoli 'Federico II' Dept of Food Science (Italy): Luca Scalfi, Paola Vitaglione, Concetta Montagnese. Ghent University (Belgium): Ilse De Bourdeaudhuij, Stefaan De Henauw, Tineke De Vriendt, Lea Maes, Christophe Matthys, Carine Vereecken, Mieke de Maeyer, Charlene Ottevaere, Inge Huybrechts. Medical University of Vienna (Austria): Kurt Widhalm, Katharina Phillipp, Sabine Dietrich, Birgit Kubelka, Marion BorissRiedl. Harokopio University (Greece): Yannis Manios, Eva Grammatikaki, Zoi Bouloubasi, Tina Louisa Cook, Sofia Eleutheriou, Orsalia Consta, George Moschonis, Ioanna Katsaroli, George Kraniou, Stalo Papoutsou, Despoina Keke, Ioanna Petraki, Elena Bellou, Sofia Tanagra, Kostalenia Kallianoti, Dionysia Argyropoulou, Stamatoula Tsikrika, Christos Karaiskos. Institut Pasteur de Lille (France): Jean Dallongeville, Aline Meirhaeghe. Karolinska Institutet (Sweden): Michael Sjöstrom, Jonatan R. Ruiz, Francisco B. Ortega, María Hagströmer, Anita Hurtig Wennlöf, Lena Hallström, Emma Patterson, Lydia Kwak, Julia Wärnberg, Nico Rizzo. Asociación de Investigación de la Industria Agroalimentaria (Spain): Jackie Sánchez-Molero, Sara Castelló, Elena Picó, Maite
Navarro, Blanca Viadel, José Enrique Carreres, Gema Merino, Rosa Sanjuán, María Lorente, María José Sánchez. Campden BRI (UK): Chantal Gilbert, Sarah Thomas, Elaine Allchurch, Peter Burgess. SIK - Institutet foer Livsmedel och Bioteknik (Sweden): Gunnar Hall, Annika Astrom, Anna Sverkén, Agneta Broberg. Meurice Recherche \& Development asbl (Belgium): Annick Masson, Claire Lehoux, Pascal Brabant, Philippe Pate, Laurence Fontaine. Campden \& Chorleywood Food Development Institute (Hungary): Andras Sebok, Tunde Kuti, Adrienn Hegyi. Productos Aditivos SA (Spain): Cristina Maldonado, Ana Llorente. Cárnicas Serrano SL (Spain): Emilio García. Cederroth International $A B$ (Sweden): Holger von Fircks, Marianne Lilja Hallberg, Maria Messerer. Lantmännen Food RED (Sweden): Mats Larsson, Helena Fredriksson, Viola Adamsson, Ingmar Börjesson. European Food Information Council (Belgium): Laura Fernández, Laura Smillie, Josephine Wills. Universidad Politécnica de Madrid (Spain): Marcela González-Gross, Jara Valtueña, David Jiménez-Pavón, Ulrike Albers, Raquel Pedrero, Agustín Meléndez, Pedro J. Benito, Juan José Gómez Lorente, David Cañada, Alejandro Urzanqui, Rosa María Torres, Paloma Navarro. 